Aus der gynäkologischen Klinik des Prof. Tauffer in Budapest.

\title{
Ein Fall von doppelter Gebärmutter mit doppelter Scheide (Uterus didelphys c. vagina duplice).
}

\author{
Von \\ Dr. Gust. A. Dirner, \\ klinischer Assistent. \\ (Miť 3 Holzschnitten.)
}

Seit dem in den letzten Decennien gemachten grossen Fortschritte in der Lehre der Entwickelung des Organismus höherer Thiere und speciell in der Frage der Missbildungen betrachten wir diese nicht mehr als blosse Naturspiele, sondern wissen es, dass sie Folgen eingetretener und der normalen Entwickelung entgegengetretener Hemmungen sind. Ueber die Natur dieser Hemmungen ist der Schleier wohl noch nicht gelüftet, aber es dürfte nicht zu lange dauern, und wir werden auch in diesem Punkte mehr Licht gewinnen. Es können die Ursachen solcher kranken Effecte im embryonalen Körper keine andere als die Ursachen der Krankheiten im erwachsenen Körper sein, nämlich physische. Ob unter diesen den mechanischen der einzige oder wenigstens erste Platz gebührt, oder ob auch thermische, elektrische u. s. w. einwirken, und weiter, ob für jede "Missbildung eine andere Hemmungsursache und für eine gewisse immer dieselbe besteht, sind Fragen, die noch präciser Entscheidung harren und zu deren Klärung es eben deshalb genauer Beschreibung aller einschlägigen Fälle bedarf, denn nur so wird es möglich, zu gewünschtem Resultate zú gelangen. 
Ich will den in der Literatur bisher verzeichneten Fällen von Eisenmann ${ }^{1}$ ), Krisse ${ }^{2}$ ), Bujalsky ${ }^{3}$, Freund ${ }^{4}$ ), Le Fort 5 , Benike ${ }^{6}$, zwei von Heppner ${ }^{7}$, Fränkel ${ }^{8}$ ), Krieger $\left.^{9}\right)$, (usco $\left.{ }^{10}\right)$, Gusserow ${ }^{11}$ ), Cooley ${ }^{12}$ ), Perrault ${ }^{13}$, Bonnet $\left.{ }^{14}\right)$, Ollivier $\left.{ }^{15}\right)$, Freudenberg ${ }^{16}$ ) und letzthin zwei von Kubasow ${ }^{17}$ ) auch einen mehrfach interessanten Fall von Uterusduplicität anreihen, der intra vitam diagnosticirt ist, aber, wenn es uns gelingt, ihn im Auge zu behalten, sein anatomisches Interesse durch Autopsie erst liefern soll.

Von den bezeichneten Fällen beziehen sich die ron Cusco, Gusserow, Freund und ein Fall Heppner's auf Uterus didelphys solidus rudimentarius; der zweite Fall Heppner's und Krieger's waren mit Atresia ani combinirt, die Kinder starben; Fränkel's Fall bei einem Monstrum diente noch als Grundlage der Aussage Kussmaul's, dass solche Uterusduplicitäten nur mit anderen schweren Abnormitäten zugleich vorkommen; Bonnet's Fall ist dunkel, war übrigens mit Ektopie der Harnblase combinirt; Eisenmann's und Krisse's Fälle sind Uebergänge zu Uterus bicornis; Bujalsky's Fall wohl auch, und zwar wurden beide Uteri nach einander schwanger, die rechte Schwangerschaft aber endete lethal; Cooley's und Perrault's Fülle sind durch Autopsie nicht erhärtet, so dass mit Sicherheit bei Erwachsenen Uterus didelphys cum va-

1) Tab. anat. quat. uteri dupl. observ. rar. sist. Argentor 1752.

2) Brit. med. Journal 1872, Vol. I, p. 571.

3) Anatomisch-pathologische Beschreibung eines Doppel-Uterus (russisch).

4) Wiener medicinische Presse 1866, 18, 3.

5) Des vices de conform. de l'utérus et du ragin etc. Paris 1863.

6) Zeitschrift für Geburtshülfe und Gynäkologie, Bd. I, S. 366.

7) St. Petersburger medicinische Zeitschrift 1870, Heft I, S. 193.

8) De organ. gener. deformitate rariss. Diss. Berolini 1825.

9) Monatsschrift für Geburtskunde $1858, \mathrm{Bd}$. XII, S. 184.

10) Gaz. des Hôpit. de Paris 1863, 22. Oet., 124, S. 494.

11) cf. Monatsschrift für Geburtskunde und Frauenkrankheiten, Bd. XXX, S. 97 .

12) M. D. Chicago III. The amer. Journ. of med, sciences 1874 , p. 515.

13) Brit. and for. med chir. Rev. from Lyon médical, Aug. 31, 1873.

14) Phil. Transaction 1725, p. 142.

15) Gazette méd. de Paris 1872,"p. 163.

16) Zeitschrift für Geburtshülfe und Gynäkologie, Bd. V, S. 334.

17) Archiv für pathologische Anatomie und Physiologie, Bd. XC, Heft 1, S. 35 u. 81 . 
gina simplice seu duplice, ohne irgend eine anderweitige Abnormitat in der Körperentwickelung und mit normalen Functionen der Geschlechtsorgane nur in den Fällen Le Fort's, Ollivier's, Benike's, Freudenberg's und in den zwei Fällen Kubasow's constatirt ist. Von diesen letzten war Freudenberg's Fall bei einer Jungfrau mit Haematometra verbunden; Kubasow's erster Fall, nur post mortem am Präparate von ihm und $\mathrm{Hor}-$ witz als Uterus didelphys erkannt, wurde bei einer Demonstration in der Gesellschaft russischer Aerzte von einer ad hoc gewählten Commission als solcher nicht angenommen; bei genauer Durchsicht der Beschreibung liegt kein ernster Grund dafür vor, wenngleich das Präparat auch kein ideales Bild eines Uterus didelphys darbietet. Sein zweiter Fall, intra vitam diagnosticirt und sehr genau beschrieben, lässt beinahe keinen Zweifel an Sicherheit, bezieht sich aber anf eine bisher sterile Frau. Unser Fall steht diesem seiner äusseren Beschreibung nach am nächsten, weil er auch eine lebende Frau betrifft; er nähert sich den Füllen Ollivier's und Benicke's insofern, als bei ihm schon ein Abortus abgelaufen ist; sein Interesse wächst aber dadurch, dass nach dem unten beschriebenen operativen Eingriffe eine Gravidität im zweiten Uterus eingetreten ist.

Unser Fall ist folgender: Im December $1881 \mathrm{kam}$ Frau M. L., 27 Jahre alt, auf unsere Klinik. Im Kindesalter war Patientin immer gesund. Sie menstruirt seit dem 14. Lebensjahre regelmässig, doch immer mit Kreuzschmerzen und sehr ausgiebig. Seit zehn Jahren verheirathet, klagte sie erst vor einem halben Jahre ihrem Arzte, es entleere sich bei jedem Beischlafe Harn von ihr. Die Untersuchung ergab, dass sie den Beischlaf nicht an entsprechendem Orte, sondern durch die Harnröhre ausübe, sowie dass sie zwei Scheiden habe, mit je einem für den Katheter passirbaren Ostium, von denen das linke nachgiebiger war.

Der behandelnde Arzt soll zu dieser Zeit das linke Ostium vaginae mit einem dreiblättrigen Dilatatorium erweitert und gerathen haben, von nun an daselbst den Beischlaf zu üben.

Patientin wurde bald schwanger, abortirte jedoch im vierten bis fünften Monate. Seitdem fühlt sie im Bauche, und besonders linkerseits, fortwährende Schmerzen und leidet auch an Fluor albus, der aber auch vorher bestand. Habituelle Stuhlverstopfung. 
Die Frau ist mittelgross, gracil gebaut, mässig gut genährt, etwas blass, mit leidendem Gesichtsausdrucke aber nicht krankhaften Aussehens. Am und im Bauche zeigt sich äusserlich nichts Abnormes; Mons veneris genügend fettreich, gut behaart, grosse Schamlippen klein, mit weniger subcutanem Fettgewebe, die kleinen Schamlippen kleiner, virginal. Clitoris normal. Aus der Harnröhre hängt ein fast wallnussgrosser, blassrother, breiter, kammartiger Wulst hervor, ein Theil der abgerissenen hinteren Harnröhrenwand. Auf seiner Oberfläche ist er epidermisähnlich ïberhäutet, bis zu dem Eingange in die Haruröhre, wo der lederartige Ueberzug in Mucosa übergeht. Wenn dieser hervorstehende Kamm in die Harnröhre zurückgeschoben wird, so haben wir bei auseinandergehaltenen Schamlippen das Bild der Atresia hymenalis vor uns, nämlich ein grosses Vestibulum ohne Ostium vaginae. Zwischen den Falten werden aber beiderseitig je ein Ostium vaginae leicht entdeckt, von denen das linke gedehnter, weiter ist, das rechte nur mit Mühe, unter Schmerzen und aus kleinen Einrissen stammender Blutung den Finger durchlässt. Hymenalgebilde sind nur an den gegen die Lippen gerichteten Theilen der Oeffnungen zu erkennen.

Durch die linke Scheide erreicht der Finger eine normal grosse Portio vaginalis mit quergestelltem Muttermunde. Bei combinirter Untersuchung findet man den dazugehörigen gänseeigrossen Uterus, vorwärts gekrümmt, auf Druck recht empfindlich und deshalb bezüglich seiner Beweglichkeit nicht genügend erforschbar. In seiner hinteren Wand ist ein subserös sitzender, haselnussgrosser Knoten zu fühlen. Die rechte Scheide ist enger, die vordere Lippe der rechten kleinen kurzen Portio vaginalis ist mit dem vorderen Scheidengewölbe verwachsen; dieser Scheidentheil ist schlanker, Muttermund klein, rundlich; die rechte Gebärmutter ist hühnereigross, von der linksseitigen getrennt zu bewegen, nicht empfindlich, anteflectirt.

Die Harnröhre lässt den Zeigefinger ohne Widerstand durch, nur der Sphincter ist geschlossen, aber auch leicht zu passiren. Durch die Blase fühlt man deutlich die getrennten. Uteri und ihren Zusammenhang in den untersten Partien. Auch die combinirte Untersuchung durch Rectum und Harnblase ergiebt getrennte Uteruskörper, zwischen den Uterushälsen aber ein ein bis zwei Finger breites, sie verbindendes membranöses Gebilde, 
welches aber ihre gegenseitige Beweglichkeit nicht hindert. Beide Ovarien sind zu fühlen. Das Septum beider Scheiden bildet einen senkrechten Keil mit nach oben gerichteter Spitze, dio schon vom zweiten Längsdrittel der Scheide an als papierdünne Membran zu den Scheidentheilen hinaufläuft. Die Basis des Keiles liegt in der Schamspalte. Das Septum ist somit bis zur Hälfte der Scheide fleischig, von dà an häutig. Beide Scheiden, vorzüglich die linke, sondern viel Schleim ab. Die Schleimhäute sind geröthet, linksseitige von blasseren Inseln durchsetzt. Harnträufeln besteht nicht.

Ohne hier eingehender die Classification der Bildungsfehler des Uterus zu würdigen, gehe ich kurz zu der uns interessirenden Anomalie, d. h. zum Uterus didelphys cum vagina duplice über, welche in unserem Falle wohl zweifellos besteht. Wir wissen es, dass sie zu Stande kommt, wenn die Müller'schen Gänge vorzeitig eine parallele Lage zu einander einnehmen und sich isolirt weiter entwickeln, wobei keine Verschmelzung stattfindet und jeder Gang zu einer Tube, einem Horn, einem Uterus und einer Scheide wird. Wir haben im strengen Sinne des Wortes nicht zwei mit allen Adnexen vollkommen versehene Uteri in der, ,doppelten Gebärmutter" vor uns, sondern nur die höher entwickelten zwei Hälften der Uranlagen der Gebärmutter, die nicht zu einem Ganzen verschmelzen konnten. Was den Stimulus zu dieser Hemmung in den ersten sieben bis acht Wochen des embryonalen Lebens abgiebt, ist noch nicht entschieden. Nach Kussmaul's ${ }^{1}$ ) und Fürst's ${ }^{2}$ ) Forschungen ist es aber nahezu sicher gestellt, dass der Eintritt der Hemmung und infolgedessen die Weiterentwickelung der. Uranlagen in paralleler Richtung, isolirt, bei unserer Anomalie noch vor die achte Woche zu verlegen ist. Bei Erforschung der Ursachen der Hemmung, welche Uterus didelphys zur Folge hat, wird deshalb der embryonale Status quo der siebenten bis achten Woche, die Lagerung der Uranlagen aller Bauchorgane u. s. w., etwaige Ernährungsstörungen, Entzündun-

1) Von dem Mangel u. s. w. der Gebärmutter. Würzburg 1859.

2) Ueber Bildungshemmungen. Monatsschrift für Geburtshülfe u. Frauenkrankheiten, Bd. XXX. 
gen, Axendrehungen, Faltenbildungen u. s. w. von höchstem $*$ Belange sein. Es ist recht wahrscheinlich, dass die Verschmelzung gehindert werde, worauf Tirsch hinweist, durch stärkere Entwickelung, längeres Persistiren und grössere Entfernung der Wolff'schen Körper von einander, welche bei der Geschlechtsdifferenzirung in weiblicher Richtung, also in der achten Woche, vergehen sollten, wie es bei dem Zusammentreffen der Uranlagen des normalen Uterus geschieht, wobei die Wolff'schen Körper unbedeutende Entwickelung erlangen und früh schwinden (Epoophoron, Paroophoron). Auch Kussmaul lässt einen Causalnexus zwischen ungewöhnlicher Grösse der Parovaria und der Doppelmissbildungen des Uterus zu. $\mathrm{Er}$ fand bei einem Uterus septus die Parovaria gross und hält die an einem Präparate von Cassan bei Uterus duplex zwischen den Tuben und Eierstöcken, an der Stelle der Parovaria gefundenen taubeneigrossen, mit syrupähnlichem Inhalte gefüllten Blasen für Hydatiden des Wolffschen Körpers.

Kussmaul zollt aber auch einer zweiten Hypothese von Krieger seine Anerkennung. Nach dieser sollte das Ligamentum recto-vesicale, ein Ueberbleibsel des Stieles der Allantois, die definitive Entwickelung des Uterus hindern. Dasselbe besteht aus zwei Bauchfellamellen, schliesst den Urachus in sich, bildet gewissermaassen das Ligamentum suspensorium uteri. Je nachdem diese Falte in einzelnen Fällen tiefer hinab steigt und sich zwischen die Uteri hineinsenkt, entstehen mehr oder weniger getheilte Uteri. - Diese Hypothese wollte ich hier deshalb in Erwähnung bringen, weil in unserem Falle im untersten Abschnitte beider Uteri ein membranöses Gebilde zu tasten war, über dessen Natur freilich bei der noch Lebenden jede Deutung unstatthaft ist. Deshalb kann ich auch auf die möglichen anderen Momentë, auf regressive und progressive nutritive Störungen der Urogenitalanlagen u. s. w. nur hinweisen, möchte mich aber der Meinung Kubasow's anschliessen, dass die Hemmungsbildungen überhaupt, und die der urogenitalen Organe speciell, Ergebnisse vieler Momente sind. Anatomisch wichtig und als Beleg zux Entwickelung des normalen Uterus für den Punkt, wo die Verschmelqung der Müller'schen Gänge beginnt, scheint mir die Gestaltung des Septum beider Scheiden, welches in der Nähe der Portiones dünn, membranös, 
an den Scheideneingängen aber voluminöser, fleischiger war, also einen Keil vorstellte mit nach oben gerichteter Spitze. Dies zeugt dafür, dass die Näherung und Verschmelzung der M üll er'schen Gänge, wie es Dohrn angiebt, im unteren Drittel beginnt, in unserem Falle auch thatsächlich begonnen haben muss und von hier nach oben und unten fortschreiten sollte, als aber auch schon die Hemmung eintrat.

Im Vergleiche mit den bisher verzeichneten Fällen möchte ich annehmen, dass auch unser Fall die Meinung Kussmaul's, dass der Uterus didelphys kein selbständiger Bildungsfehler wäre, ausschliesst und diesbezüglich dem zweiten Falle $\mathrm{Ku}$ bas ow's ganz congruent ist. Weiterhin ist es bemerkenswerth, dass beide Uteri nicht gleichmässig entwickelt sind und hier, wie in den Fällen Ollivier's, Kubasow's, Benike's, Bujalski's, der linke der grössere ist. Ich darf dies wohl trotz der im linken Uterus bis zum vierten bis fünften Monate bereits abgelaufenen Schwangerschaft annehmen, da die rechte Portio vaginalis bedeutend kleiner ist als die linke. Die normalen Menstruationen in unserem Falle sind aller Wahrscheinlichkeit nach von beiden Hälften verrichtet. $\mathrm{Ob}$ beide Uteri abwechselnd oder zugleich menstruiren, ist leider zu beobachten versäumt worden; wir haben aber die Aussicht, das Versäumte nachholen und dann mittheilen zu können. Nach dem unten näher beschriebenen chirurgischen Eingriffe stand einer Schwangerschaft im rechten Horne kein Hinderniss mehr im Wege. Die Empfängniss ist seitdem auch wirklich erfolgt.

Ich übergehe die nähere Beschreibung unserer erfolglosen Bemühungen, die verschiedenen oben erwähnten Leiden der Kranken zu beseitigen. Sie wichen erst, als wir uns, auch auf dringenden Wunsch der Kranken selbst, zur chirurgischen Operation entschlossen.

Es war die Vereinigung beider Scheiden, ferner die Excision des urethralen Tumor und die Verengerung der klaffenden Urethraöffnung angezeigt, ebenso sehr von ästhetischem Standpunkte aus als auch um einem möglicherweise eintreffenden Harnträufeln entgegen zu treten.

Diese Operationen hat Prof. V. Tauffer am 19. April 1882 folgendermaassen ausgeführt:

In Narkose wurde das Septum durch zwei, von beiden Schei- 
den aus in Keilform geführte Schnitte von seinem unteren Ende, respective der hinteren Scheidewand losgetrennt, und zwar hinauf bis in die Nähe der Portio vaginalis, wo das papierdünne Septum $1-11 / 2 \mathrm{~cm}$ lang stehen gelassen, weil es nicht rathsam schien, bei Unkenntniss der Faltenverhältnisse des Peritoneum zwischen und an den beiden Uteri mit dem Messer hinauf zu greifen. Die Wundränder der auf der hinteren Scheidewand so entstandenen Wunde wurden im unteren, fleisehigen Theile des Septum mit Silberdraht vereinigt, wodurch das auf Fig. 3 in der Richtung $a d b$ mit unterbrochener Linie angemerkte Verhältniss entstand. Auf dieselbe Art, aber mit nach oben gerichtetem Keile, wurde das Septum von der vorderen Scheidewand abgetragen und die Wundränder auch mit Silberdraht vereinigt. Die Entfernung des aus der Harnröhrenöffnung heraushängenden Wulstes und die Verengerung der Urethra geschah folgendermaassen: In der hinteren Wand der weit offenen Urethra wurden unter spitzem Winkel zwei halbmondförmige, symmetrische Schnitte geführt, deren Sicheln gegen die Mittellinie schauen und den Wulst umgebend im Punkte $h$ des Introitus zusammentreffen (Fig. 3 $e f h g e$ ). Nach Entfernung des Wulstes und Vereinigung der. Wundränder in der Nahtlinie e $i$ h war das Lumen der Urethra nahezu auf das Normale gebracht. Dieser Eingriff verlief reactionslos, per primam. Die Drähte wurden am zehnten Tage entfernt und die Kranke geheilt entlassen. 

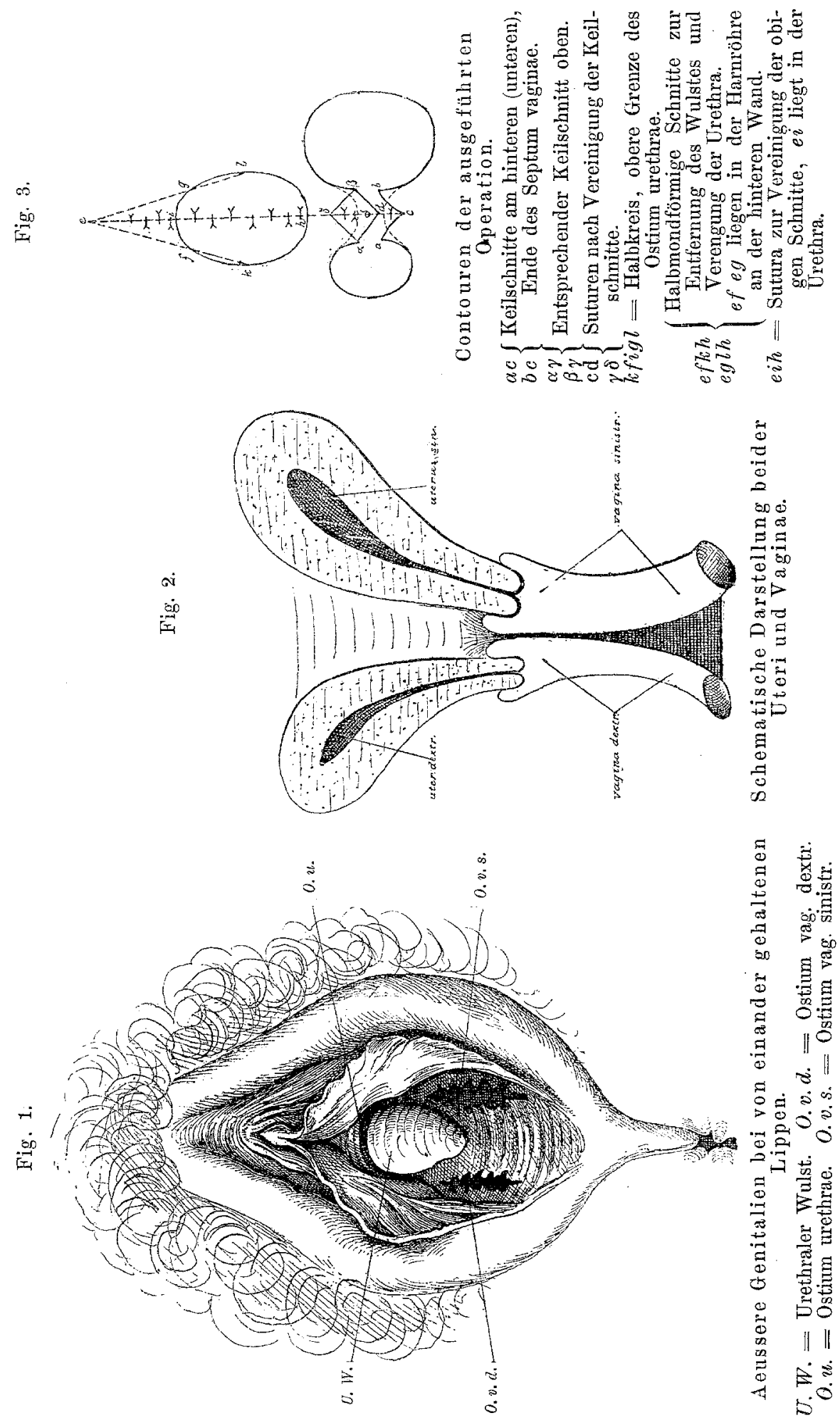
Nachdem sie im Mai 1882 unsere Klinik verlassen, wurde sie schwanger, abortirte aber wieder im vierten bis fünften Monate (October), angeblich aus dem linken Uterus. Während dieser Schwangerschaft hatte sie keine Menses.

Der Abortus verlief ohne Complication. Nach drei Wochen stellte sich eine Blutung ein, die zwei Wochen währte. Darauf kamen die Menses in vier bis fünf bis sechs Wochen ohne Schmerz und in kleinerem Maasse. Ihre letzte Menstruation hatte die Frau im Februar (16.) 1883. Sie spürte die Schwere anfangs wieder links, später rechts. Seit Mitte Juli Kindesbewegungen. Befinden gut.

Status praesens am 18. November 1883: Die Frau ist bedeutend fetter geworden; der Fundus uteri reicht drei Querfinger unter den Processus xyphoideus, vorliegender Theil Kopf, Herztöne rechts. Aeussere Genitalien bräunlich verfärbt; hintere Vaginalwand drängt sich stark hervor; Urethralöffnung zwischen Falten versteckt.

In der Mitte der Vagina eine Scheidewand, weich, aufgelockert anzufühlen; davon rechts das Scheidengewölbe durch den vorliegenden Kopf tief herabgedrängt, die Portio darin aufgelöst, Muttermund 11/2 Querfinger weit, dünn, weich. Schädel liegt in zweiter Lage tief im Eingange. - Links ist die Wölbung: des Schädels auch durchzufühlen; linke Portio fast platt an dic Beckenwand gedrückt, Muttermund lässt die Fingerspitze durch; linker Fundus uteri in der Höhe und hart in der Nähe der linken Spina superior anterior zu fühlen; Corpus weicher, dicker, an der linken Wand des schwangeren Uterus auf und ab zu bewegen.

Die Wehen begannen am 18. November, Nachmittags 4 Uhr; die zu der Zeit vorgenommene Untersuchung ergab zweite Schädellage, bei für einen Finger durchgängigem Muttermunde und verstrichenex Portio und Cervix; die Wehen waren selten, schwach, setzten einen halben Tag ganz aus, und wurden erst nach 29 Stunden vom Beginn gerechnet bei zwei Querfinger breitem Muttermunde intensiver, so dass nach $4{ }_{2}^{1}$ Stunden der Muttermund verstrichen war, die erste Geburtsperiode also $33^{1} / 2$ Stunden dauerte. - Nach 21/4 Stunden gebar die Frau am 20. November früh 1\%/2 Uhr ein lebendes, reifes Mädchen von $2600 \mathrm{~g}$ Gewicht und $46 \mathrm{~cm}$ Länge, Kopfumfang $34 \mathrm{~cm}$. - (Beckenmaasse: Spinae 
25, Cristae 27, Conjugata externa 20.) Die Placenta wurde nach fuinf Minuten ausgestossen.

Sogleich nach der beendeten Geburt untersuchte ich die Frau und fand den Geburtskanal völlig unverletzt; das Septum im oberen Theile der Vagina ohne geringsten Einriss. - Mein Hauptinteresse concentrirte sich auf die linke Gebärmutter und die Verbindung mit der rechten. Linke Portio ist weich, locker, saftreich; Os externum für einen Finger leicht durchgängig; Corpus steht gerade, fast in der Höhe des Nabels, ist leicht zu bewegen; bei combinirtem Handgriffe durch Vagina und Bauchdecken treffen beide Hände zwischen beiden Uteri sicher aneinander, so dass zwischen diesen nur eine dünne, ligamentöse Verbindung anzunehmen ist, die aber eine freie gesonderte Beweglichkeit erlaubt.

Es war wiederholt gut zu fühlen, wie der linke Uterus unter den Fingern, bei Contraction des rechten Uterus, auch ganz hart und kleiner wurde, dann wieder erschlaffte. Das linke Ovarium war in normaler Lage und Grösse gut zu fühlen.

Das Wochenbett war ein einziges Mal am fünften Tage Nachmittags von einer $38,1^{\circ} \mathrm{C}$. Temperatur gestört, ohne sonstige objective und subjective Erscheinungen; die Wöchnerin stand am zwölften Tage auf und hat am 17. unser Institut verlassen.

Die Untersuchung ergab am 6. December: Septum vaginae als sichelförmige Falte unversehrt erhalten; rechte Portio kurz, Ostium externum querstehend, bohnengross, Corpus anteflectirt, leicht aufzustellen, frei beweglich, beinahe normal involvirt und schmerzlos; rechtes Ovarium nicht gefunden, auf Druck in dieser Gegend recht empfindlich.

Linke Portio schlank, länger; Muttermund nimmt die kleine Fingerspitze auf, Corpus kleiner als das rechte, daran jetzt kein Fibromknoten zu fühlen. Linkes Ovarium leicht und sicher zu. finden. Keine Schmerzhaftigkeit.

Es hat unser Fall an Interesse nun bedeutend gewonnen. Es ist durch genaue Untersuchung nach der Geburt, also bei geeigneten Verhältnissen, erhärtet, dass die Trennung der Hörner eine vollständige ist, dass ihre Verbindungsfläche nachgiebig sein muss und nur auf den untersten Theil beschränkt, denn die gesonderte Beweglichkeit ist auffallend gross. Es ist wahrscheinlich, dass beide 
Uteri nach einander schwanger waren, denn bisher war der linke entschieden grösser, der rechte virginal; jetzt nach Involution im 17. Tage, der rechte bedeutend grösser als der linke, der jetzt etwas kleiner erscheint als bis vor dieser Schwangerschaft. Es ist auch wahrscheinlich, dass beide vorangegangenen Schwangerschaften im linken Uterus abgelaufen sind, wo sie im fünften Monate unterbrochen wurden. Den Grund des Abortus kennen wir nicht; es hat sogar die Wahrscheinlichkeit, dass ihn ein Fibromknoten verursachen konnte, nicht zugenommen, denn ich kounte bei der letaten Untersuchung diesen angedeuteten Fibromknoten mit Sicherheit nicht mehr finden.

Sicher und genau beobachtet ist nun aber die letzte, die normal verlaufene Geburt aus dem rechten Uterus, der bei den jungen Eheleuten bald neue folgen können, die wir - wenn die Frau ihr Versprechen hält - auch selbst zu leiten die Aussicht haben. 